\title{
INNOVACIÓN Y TECNOLOGÍA: NECESIDAD DE CAMBIOS EN LOS PROCESOS DE ENSEÑANZA - APRENDIZAJE, INCLUSIÓN DE COMPETENCIAS Y COMPROMISO DESDE LA FORMACIÓN DOCENTE
}

Javier Rodríguez-Torres': Universidad de Castilla - La Mancha. España javier.rtorres@uclm.es

\section{RESUMEN}

Coincidimos con Fullan al considerar que una de las principales dificultades para la mejora de las escuelas de occidente no es la ausencia de innovación, más bien la presencia de demasiados proyectos novedosos inconexos, episódicos, fragmentados y adornados de forma superflua. El mayor problema al que se enfrentan las escuelas es la fragmentación (balcanización) y exceso de innovaciones. Los cambios estructurales, de organización, de gestión, de diseño curricular, de condiciones laborales, de tipología de alumnado... que vivimos en los últimos veinte años son, cuando menos, asombrosos y productores de gran INCERTIDUMBRE, se suma, además, la realidad regional, es decir, la propia configuración de nuestro país. Por otro lado, no podemos negar las posibilidades de acceso al saber que proporcionan los artefactos tecnológicos, produciendo un efecto psicológico de engrandecimiento que eleva nuestra autoestima y estimula a desarrollos ilimitados. Ahora bien, sabemos que cuando una actividad realizada con TIC se centra en la máquina y aplicaciones, descontextualizadamente, no genera más que información. La euforia y utilización acrítica de la tecnología como innovación, puede esconder un nuevo mesianismo y encubrir una perversión ideológica: la nivelación ilusoria de la humanidad como consecuencia de la nivelación potencial de un grupo de individuos, de una parcela de sus vidas en el ciberespacio.

PALABRAS CLAVE: Innovación - Tecnología - Competencias - Formación de profesorado - Paradigmas

\footnotetext{
${ }^{1}$ Autor correspondiente

Javier Rodríguez-Torres: Universidad de Castilla - La Mancha. España

Correo: javier.rtorres@uclm.es
} 


\title{
INNOVATION AND TECHNOLOGY: NEED OF CHANGES IN THE PROCESSES OF EDUCATION - LEARNING, INCORPORATION OF COMPETITIONS AND COMMITMENT FROM THE EDUCATIONAL FORMATION
}

\begin{abstract}
We agree with Fullan that one of the most important difficulties for the improvement of the schools of the rich western countries is not the absence of innovation, but rather the presence of too many new unconnected, projects, fragmented and adorned with superfluous forms. The major problem that the schools face is fragmentation and excess of innovations. The structural changes of organization, of management, of curriculum development, of working conditions, of typology of student body and so on. The last twenty years are, amazing and cause great UNCERTAINTY. To this situation we must add, the regional reality, that is to say, the own configuration of the country. On the other hand, we cannot deny the possibilities of access to knowledge provided by the technological appliances and that causes, producing a psychological effect aggrandizement that increases our selfesteem and stimulates us to unlimited developments. We know that when an activity carried out with TIC is focused on the computer way and its applications, moreover these applications are not contextual and only generates information. The euphoria and uncritical use of technology as an innovation can hide a new messianic and to conceal an ideological perversion: the illusory levelling of the humanity as a consequence of the potential levelling of a part of their lives in cyberspace.
\end{abstract}

KEY WORDS: Innovation - Technology - Competitions - Educational formation Paradigms

\section{INTRODUCCIÓN}

Cada persona es un agente de cambio (Fullan).

¿Debe cambiar la Escuela?, ¿Cómo?, ¿Cuánto?, ¿Merece la pena tanto esfuerzo?, ¿Hay algo por lo que merezca la pena luchar en la escuela? 


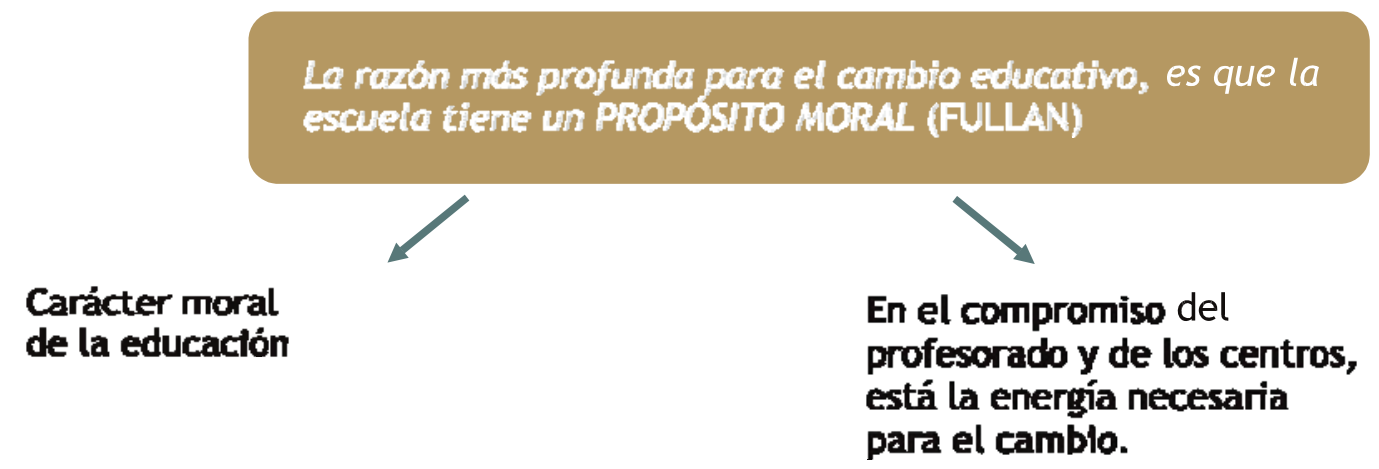

Figura 1. Razones del cambio educativo

Nadie discute que las TIC suponen un cambio, pero casi nadie habla de que este cambio tiene que ir acompañado de una nueva semiótica de la escuela. Estamos incorporando las TIC en espacios concebidos para una enseñanza. Las aulas deben concebirse como entornos de trabajo. La innovación, en la actualidad, va asociada a la tecnología. La Administración debe proporcionar los equipos necesarios, velar por su mantenimiento y renovación. Además, de desempeñar un papel de liderazgo.

Pero, compartimos con otros autores, que la introducción de cualquier medio tecnológico en los centros educativos pasa ineludiblemente por disponer de una actitud convencida y favorable por parte del profesorado, como por una adecuada formación del mismo para su correcta incorporación en su práctica profesional (Cabero, Duarte y Barroso, 1998). Es la pieza clave para que tenga éxito cualquier innovación en la enseñanza. Por tanto, en este nuevo contexto tecnológico es necesario que el profesorado cambie su mentalidad, su actitud y su rol con respecto a la enseñanza.

\section{METODOLOGÍA}

La presente investigación emplea como técnica científica el método análitico-sintético, mediante el cual se descompone un objeto, fenómeno o proceso en los principales elementos que lo integran para analizar, valorar y conocer sus particularidades, y simultaneámente a través de la síntesis, se integran vistos en su interrelación como un todo. 


\section{ANÁLISIS Y DISCUSIÓN}

\subsection{A vueltas con la innovación educativa}

A poco que se reflexione, máxime cuando se está inmerso en alguna comunidad educativa, se concluye, sin dudar, que el éxito de cualquier innovación en el ámbito educativo depende en gran medida de toda esa comunidad ${ }^{1}$ y en especial de la actuación del docente que, a su vez, viene determinada, sobre todo, por su formación. Por ello, la integración curricular de las TIC, requieren, una adecuada planificación y formación.

Compartimos con Salinas (Salinas, 1999: 6) Los cambios en educación, a cualquier escala, para que sean duraderos y puedan asentarse, requieren que cualquier afectado por dichos cambios entienda y comparta la misma visión de cómo la innovación hará que mejore la educación: profesores, administradores y la comunidad educativa entera deben estar involucrados en la concepción y planificación del cambio desde el primer momento.

Resulta ilustrativo lo planteado por Hargreaves de cómo deberían ser implementadas políticamente las innovaciones, aludiendo fundamentalmente a la necesaria participación, de forma directa, del profesorado en el cambio, y aunque posteriormente profundizaremos en esta cuestión, valgan las palabras de este autor para sintetizar lo que acabo de expresar:"Una reforma de arriba-abajo, sin una innovación de abajo hacia arriba, no creará, las escuelas que necesitamos para el mundo de mañana...." (Hargreaves, 1999:55).

Sabemos que el término innovación es polisémico, comenzaremos dando una respuesta a la cuestión que nos surge sobre su concepto.

Siguiendo a Tejada (Tejada, 1998) innovación representa una gama variada de significados desde las referencias sustantivas (qué, por qué y para qué innovar) hasta referencias de tipo procesual (cómo, condiciones, mecanismos...). Entre otras acepciones encontramos:

Acción y efecto de innovar. RAE, consulta electrónica 05 - 10 - 11

a) Acción permanente realizada mediante la investigación para buscar nuevas soluciones a los problemas planteados en el ámbito educativo. (Diccionario de las ciencias de la educación, 1987: 796)

b) Una innovación en educación es un mejoramiento medible o evaluable que sea fruto de un proceso deliberado y logre mantenerse durante cierto tiempo. (Huberman, 1973, citado por Latapi y cols. 1997)

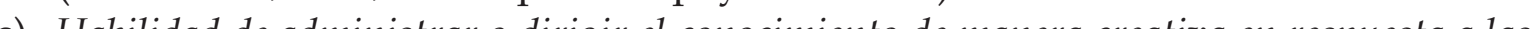


Resulta muy aclaradora esta definición de lo que nos advierte (Hargreaves, 2001) como $<<$ nueva ortodoxia educativa $>>$ que no es otra cosa que proyectar en la educación, al amparo de la ideología neoliberal, planteamientos similares a los que mueven la empresa y que bajo ese discurso de $<<$ calidad educativa $>>$ se justifican planteamientos de privatización como tendencia de los actuales responsables políticos.

De estas definiciones se deriva que la innovación tiene que ver con una actividad sistemática y consciente, que trata de dar respuestas a carencias o déficits en el ámbito educativo.

No obstante, todas ellas son bastante asépticas, y dejan sin tratar la importancia y relevancia que el campo de la innovación como ámbito de estudio ha tenido en las dos últimas décadas.

çSi nos acercamos a este campo de estudio veremos que ninguna de ellas hace hincapié en la innovación ligada al cambio como un proceso complejo, incierto, no lineal, y ligado al desarrollo profesional del docente y su compromiso con la mejora de la educación (Fullan, 1992). Y lo que es más importante, que el concepto de innovación y los modos en que se lleven a cabo, vendrán determinados por el tipo de racionalidad que informe su sentido, las finalidades que persiga, los procedimientos que se arbitren y el papel del profesorado en todo ello.

Nosotros, siguiendo a J. Carbonell y como definición bastante aceptable y aceptada, según el mismo autor, proponemos Innovación como:

Serie de intervenciones, decisiones y procesos, con cierto grado de intencionalidad y sistematización, que tratan de modificar actitudes, culturas, contenidos, modelos y prácticas pedagógicas. Y, a su vez, de introducir, en línea renovadora, nuevos proyectos y programas, materiales curriculares, estrategias de enseñanza y aprendizaje, modelos didácticos y otra forma de organizar y gestionar el currículum, el centro y la dinámica del aula. (Carbonell ,2002:17).

Desde esta definición podemos afirmar que la innovación en el ámbito escolar es consustancial al reto de los más inquietos y casi siempre comprometidos con proyectos ideológicos de signo progresista, en los que la productividad no pasa de ser una preocupación marginal (Boumard, 2001, tomado de San Martín, 2004: 134135).

La innovación en el contexto educativo se asocia a renovación, cambio y a mejora, aunque toda innovación supone renovación, cambio y mejora; sin embargo, no toda renovación, cambio y mejora es innovación. 
que implica el cambio en la práctica y del desarrollo de las estrategias correspondientes para producir reformas ventajosas" (Fullan, 1992:7).

Para este autor, que representa una línea de investigación y estudio importante en relación con la innovación y el cambio en la escuela, hay tres condiciones en torno a las cuales debe pivotar toda innovación.

a) Aprendizaje de nuevos recursos y materiales.

b) prácticas docentes.

c) concepciones y creencias.

Y todo ello, articulado en torno a dos dimensiones para él básicas, que inciden en la implementación y mantenimiento de las innovaciones: la dimensión intelectual del cambio, y la dimensión moral. Esto nos lleva a reflexionar sobre cuándo hay innovación.

Siguiendo, nuevamente, a J. Carbonell (Carbonel, 2002: 19 - 20) los factores de innovación que él expone, los trascribimos a continuación, advirtiendo al lector / a que no siempre se producen a la vez, que se suceden, en ocasiones, con timidez y otras con toda fuerza y evidencia. 


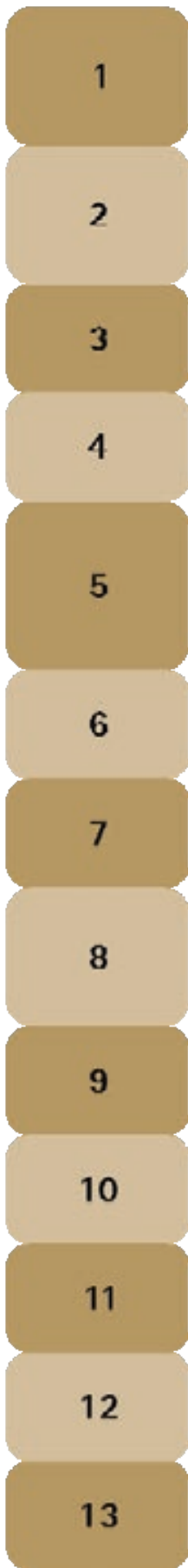

El cambio y la innovación son experiencias personales que adquieren un significado particular en la práctica, ya que aquélla debe atender tanto a los intereses colectivos como individuales.

La innovación permite establecer relaciones significativas entre distintos saberes de manera progresiva para ir adquiriendo una perspectiva más elaborada y compleja de la realidad.

La innovación trata de convertir las escuelas en lugares más democráticas, atractivos y estimulantes.

La innovación trata de de provocar la reflexión tedrica sobre las vivencias, experiencias e interacciones en el aula.

La innovación rompe con la clásica escisión entre concepción y ejecución, una división propia del munda del trabajo y muy arraigada la escuela mediante el saber experto y el "no saber" del profesorado, mero aplicador de las propuestas y recetas que le dietan

La innovación amplia el ámbito de autonomla pedagógica que no socioeconómica de los centros y del profesorado

La innovación apela a las razones y fines de la educación y a su continuo replanteamiento en función de los contextos especlficos y cambiantes

La innovación no se emprende nunca desal aislamiento y la soledad sino desde el intercambio y la cooperación permanente como fuente de contraste y enriquecimiento.

La innovación trata de de traducir ideas en la práctica cotidiana, pero sin olvidarse nunca de la teorla, conceptos ambos indisociados.

La innovación hace que afloren deseo, inquietudes e inteneses ocultos o que habitualmente pasan desapercibidos en el alumnado.

La innovación facilita la adquisición del conocimiento, perc también la comprensión de lo que da sentido al conocimiento.

La innovación es conflictiva y genera un foco de agitación intelectual permanente.

En la innovación no hay instrucción sin educación, algo que, quizá por abvio y esencial, se olvida con demasiada frecuencia.

Figura 2. Factores de la innovación 
Desde nuestro punto de vista, para que realmente exista innovación, es necesario un constante diálogo con los profesores y que entiendan la esencia de la innovación y qué implicaciones tendrá en su práctica cotidiana. Queremos decir, hay que impulsar la reflexión crítica y deliberativa del profesor con respecto a la innovación que se quiera poner en práctica (Gallego, 1994).

\subsection{Modelos en la promoción de la innovación}

\section{Modelo tecnológico}

Siguiendo a Bautista (Bautista, 1994) surge del paradigma científico y utiliza el método experimental. Considera la innovación (conducida por investigadores expertos) como un valor absoluto. Se considera que la tecnología es éticamente neutral; es simplemente un medio, producto de la aplicación de la ciencia, y debe estar libre de interferencias psíquicas (emociones...) y sociales (ideologías...). Otro presupuesto es el determinismo tecnológico. A partir de evaluaciones de costes, riesgos y beneficios, se realizan las innovaciones que resultan del interés de las multinacionales. Se tiende a mantener el orden patrimonial y jerárquico existente.

Las innovaciones promovidas por las Administraciones Educativas e implantadas a nivel general no logran en muchas ocasiones sus objetivos, aunque cuenten con el respaldo de eminentes especialistas que prescriben buenas prácticas, lo que hay que hacer y lo que el profesor hace y proponen al profesorado cambios curriculares que buscan la eficacia, la excelencia, la calidad,... sin tener en cuenta la especificad de cada centro, de sus profesores y de sus alumnos. Nos sumamos a la idea de San Martín (Martín, 2004:133) “... en estos momentos términos como <<calidad >>, <<escuelas eficaces $>>$,... tienen mucha mayor presencia en el discurso pedagógico dominante que el de la innovación"

Este modo de entender la innovación en los centros sigue, de algún modo, vigente en la actualidad y es el fundamento de nuestras recientes reformas educativas. Han llegado coreadas de materiales institucionales (libros blancos, cajas rojas,...) poco utilizados en los centros, pero muy bien explotados por la industria editorial que, con sus <<expertos>> facilitan su consumo y digestión en los centros. En la formación del profesorado se ha profundizado en los materiales creados por esos <<expertos >> que han protegido a su obra y a su empresa. Entienden, como conclusiones, que el profesorado dispone sólo de experiencia y práctica poco elaborada y que su función es la de <<aplicador >> de las supuestas propuestas innovadoras, de los supuestamente neutrales expertos.

En este sentido Carr v Kemmis (Carr \& Kemmis, 1988) aroumentan aue el nuevo 
instituciones educativas con discursos que producen nuevas formas de normalizar las relaciones de poder, Foucault (Foucault, 1970: 46) nos lo expresa así: "Cada sistema educativo es un medio político de mantener o modificar la adecuación de los discursos con el conocimiento y el poder que traen consigo".

Reinterpretando a Nieves Blanco, la situación actual con respecto a las reformas educativas impulsadas desde políticas neoliberales y globalizadoras, lo sintetizamos en el siguiente esquema que hemos elaborado.

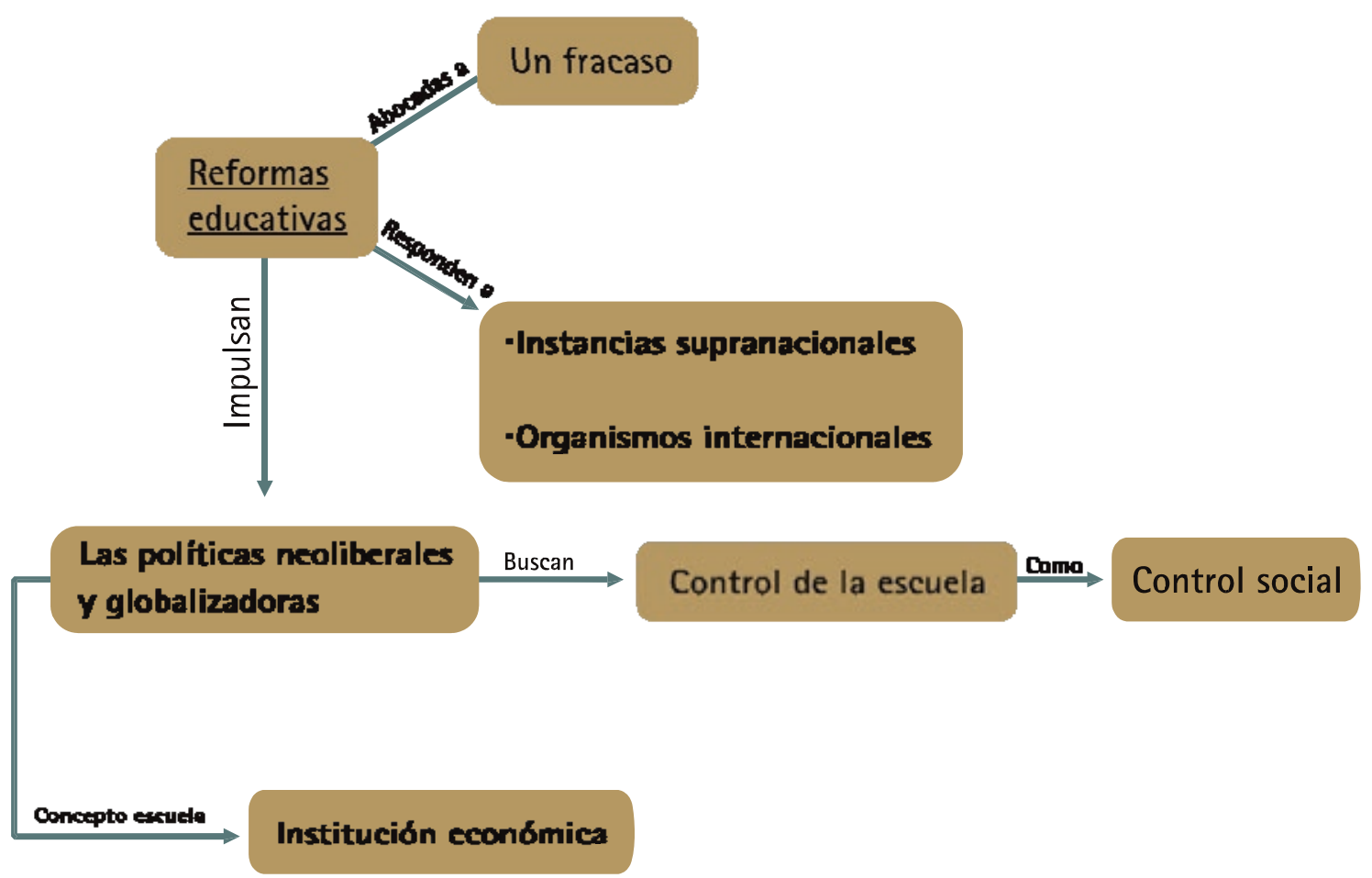

Figura 3. Reformas educativas y políticas neoliberales

\subsection{Modelo práctico o situacional}

Siguiendo de nuevo a A. Bautista (1994) y situado ahora este autor en el paradigma crítico se detiene en analizar una serie de rasgos que definen la innovación educativa y que pasamos a señalar a continuación y partiendo, del pensamiento pedagógico construido por Stenhouse, Elliot, Freire, Kemis, Giroux, Huberman, Fullan, Hargreaves, entre los más destacados, que nos revelan: 
el proceso de enseñanza y aprendizaje, esto es, la importancia subjetiva del cambio. Fullan a este respecto nos dice que el uso de nuevos materiales y la introducción deplanteamientos curriculares innovadores o de las últimas tecnologías sólo es la punta del iceberg: las dificultades están relacionadas con el desarrollo por parte de los profesores de nuevas destrezas, comportamientos y prácticas asociadas al cambio, así como con la adquisición de nuevas creencias y concepciones vinculadas al mismo.

b) La reivindicación del concepto innovación para las prácticas y éticas cotidianas.

c) La necesaria sostenibilidad en el tiempo que debe esperarse de dichas innovaciones.

Estos rasgos los contempla Fullan que al respecto nos dice que la concepción sobre innovación, de forma general, busca el bien de todos los afectados por un problema, que deben de estar presentes y participar en el análisis de alternativas y en la toma de decisiones: democratización y descentralización. De forma más pormenorizada podemos decir que se fundamenta en dos claves:

a) El cambio en la enseñanza y aprendizaje pasa por un cambio en las concepciones, actitudes y rutinas del profesorado

b) Prácticas de trabajo colaborativo sobre la reflexión de la propia práctica y aprendizajes ${ }^{7}$ permitiendo "deconstruir de manera crítica las formas de presentación de la realidad que se ofrecen como dadas y objetivas" (Hernández, 1999:44). 


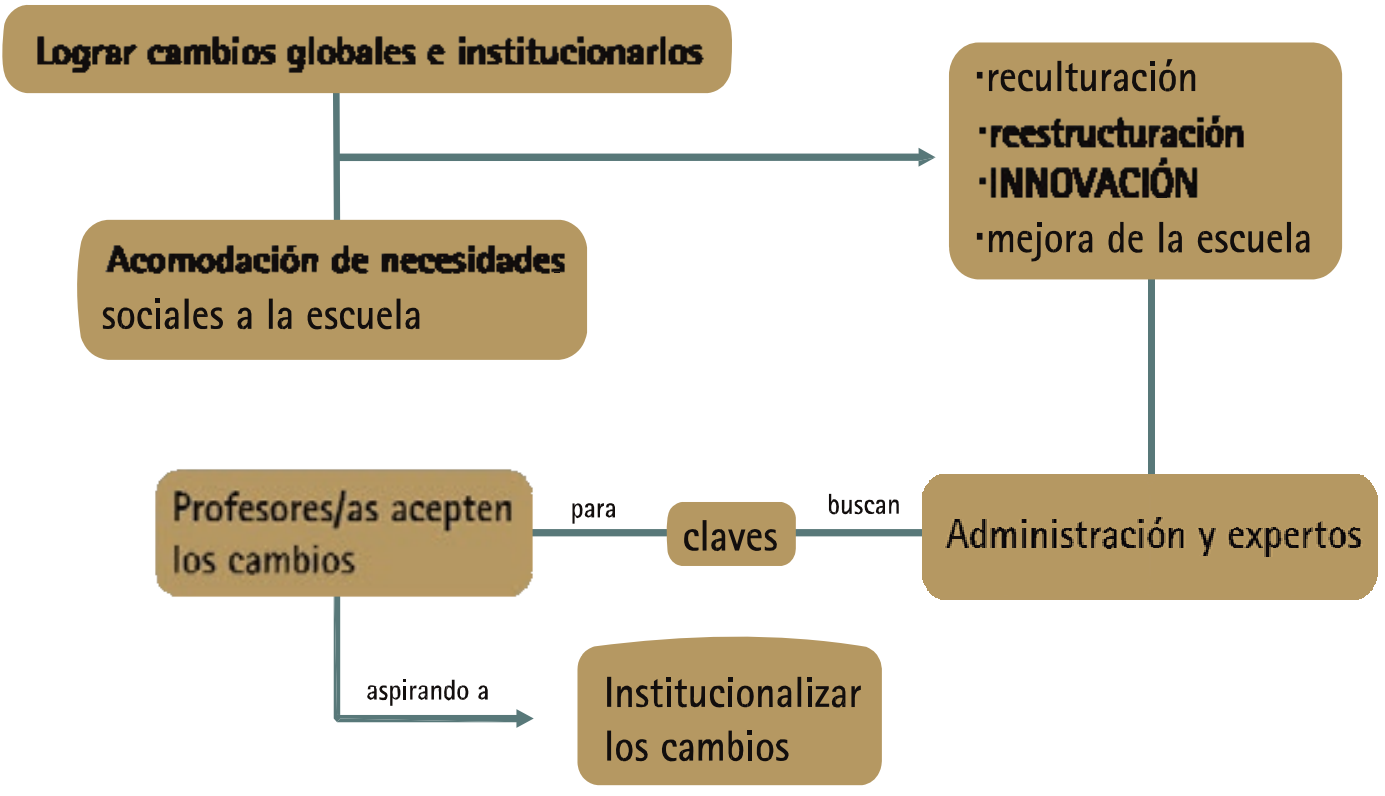

Figura 4. Institucionalización de cambios

De forma sintética, la consideración de cada uno de los contextos particulares alumnos, cultura de centro, Proyecto Educativo, intereses y desarrollo profesional de los docentes en los que se quiere desarrollar la innovación, y especialmente la consideración del profesor (sus creencias, experiencias, conocimientos $^{8}, \ldots$ ) como mediador e investigador en el aula que reflexiona sobre su propia práctica, nos abre nuevas perspectivas para lograr una innovación educativa en la que implique y participe de forma colaborativa y democrática todos los integrantes del centro.

Desde esta perspectiva y siguiendo a autores, para nosotros de referencia,(Blanco, 2001), (Carr \& Kemis, 1988), (Gimeno, 2001, 2005, 2006), nos encontramos con algunas claves para actuar facilitando la transformación de la escuela, planteamientos coherentes con la idea de innovación que venimos defendiendo. Exponemos dos mapas conceptuales, fruto de esta rica bibliografía, que resultan clarificadores en dos sentidos y para dos contextos interrelacionados: 
a) en la propia escuela

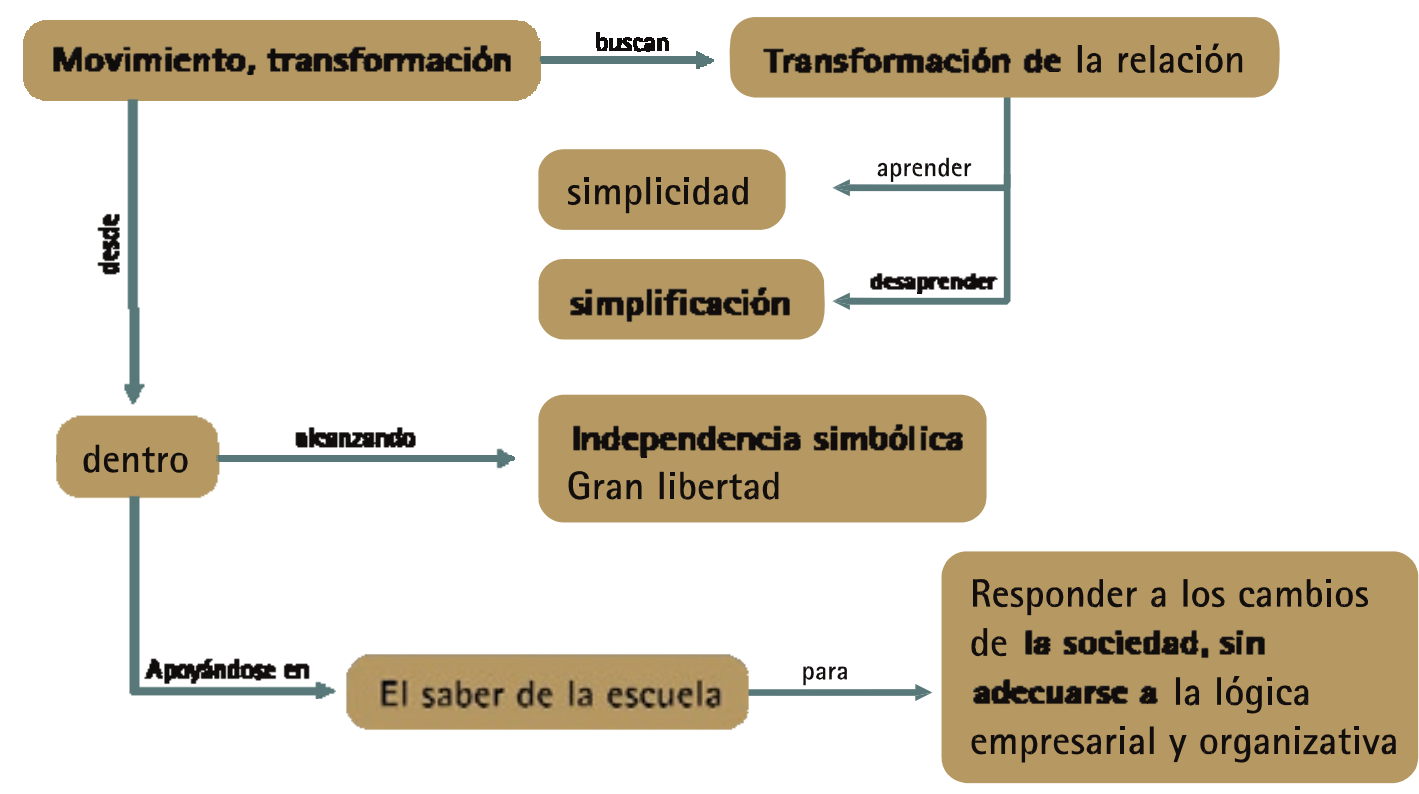

Figura 5. Transformación en la escuela

b) en la investigación educativa

\section{Investigación educativa}

\section{- contribuir a desbelar}

la saviduria, sin

someterse a códigos

externos

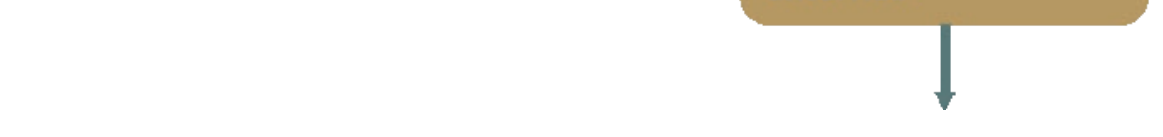

\section{PODER*}

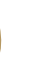

4

Relaclones de autorldad

\section{Maestros/as}

- Poder en el sentida que otorga en beneficio de la sociedad

Foucault, esto es, poder en como ejerce la gente el conocimientc para interve nir

Figura 6. Transformación en la investigación educativa

\subsection{Las TIC como innovación}

Epistimológicamente, resulta claro que la enseñanza se orienta por un tipo de conocimiento poco normativo; por el contrario, en el campo de la tecnología, sucede 
Es importante comenzar con esta idea, porque la innovación se puede instrumentalizar en la educación haciendo que se haga uso de estrategias y procedimientos que orienten las actuaciones fundamentalmente del profesor, a la consecución de los resultados previstos de antemano. Esto desvirtúa lo dicho con anterioridad con respecto a innovación. El peligro de asociar la innovación a un proceso tecnificado suele maquillarse con la asociación que se establece entre la aplicación de innovación como algo nuevo, aunque en la práctica y en la realidad concreta, se convierte en hacer "más de lo mismo". Al respecto son relevantes las palabras de San Martín cuando nos advierte de:

Mientras en la enseñanza la innovación da cabida a prácticas distintas, incluso contradictorias o a veces recuperadas del pasado, en el caso de las TI la innovación representa un estado de necesidad para ofrecer algo nuevo y original, para diseñar artefactos que en alguna característica sean mejores que los precedentes. (2004:134).

A pesar de las proclamas apocalípticas que relacionan a las TIC con la deshumanización de la escuela y la sustitución del profesorado por estos artefactos, pedagógicamente no podemos sustraernos a ellas en cada uno de los centros. Castells (Castells, 1997) nos cuenta cómo se modifican los espacios publico y privado con los flujos de información, y de los nuevos tiempos (formas) laborales, vitales. Del mismo modo, indica la creciente distancia entre globalización e identidad o entre la red y el yo; y el aumento de las desigualdades sociales y culturales al quedar excluidos de las redes de información $<<$ brecha digital $>>$.

Sin embargo, tampoco hemos de esperar, -como algunos anuncian, fruto de intereses mercantilistas y de poder-, que llenar la dotación en las aulas de artefactos sofisticados produzca un cambio en la enseñanza, tal y como lo hemos expresado en el primer apartado. Cuestión importante por la presencia dominante de un discurso que, como venimos señalando, asocia tecnología a innovación y la innovación al cambio.

Comprobamos, por nuestra propia experiencia que lograr un cambio de actitud de los profesores es difícil y complejo, pero es lo consustancial para que estos recursos generen cambios significativos en la escuela. Distintos autores señalan la formación permanente del profesorado, en este campo, en la modificación de sus actitudes, creencia; así como entender que las TIC no son artefactos mágicos que generan innovación por si misma, sino que lo participan en ella.

Las tecnologías amplían y enriquecen las capacidades humanas, pero no nos nronorcionan lo aue no tenemos. como hemos visto. generar cambio. entendido 
Estas reflexiones implican un nuevo perfil de profesor, que sigue siendo clave en la educación, capaz de asimilar y sumir cambios constantes en sus funciones y tareas en la medida que los cambios socioculturales generen nuevas demandas educativas. Cuestión sobre la que nos hemos detenido en el capítulo anterior.

Al respecto J. Adell (Adell, 1997:15) nos plantea este nuevo perfil docente que "la misión del profesor en entornos ricos en información es la de facilitador, la de guía y consejero sobre fuentes apropiadas de información, la de creador de hábitos y destrezas en la búsqueda, selección y tratamiento de la información".

Todo esto implica un cambio que debe tener su particular traducción en los programas de formación permanente, en el trabajo con las TIC, tomando como referencia dos dimensiones fundamentales, a saber:

1. Las actitudes que influirán en la introducción curricular, superando esa dicotomía tecnofóbicos vs. tecnofílicos

2. Las saberes que faculten al docente para cumplir una serie de roles en relación con:

a) alumnos: facilitador, orientador, creador de ambientes y trabajo colaborativo

b) práctica docente: adquirir mayor compromiso en torno a las TIC, creación de sus propios materiales

De la bibliografía consultada surge el siguiente cuadro, que intenta reflejar los posicionamientos de distintos autores con respecto de éste, a las competencias que tiene que tener un docente en TIC. Del mismo y de forma general, podemos extraer algunas ideas entorno a la necesidad de modificar rol del docente, el profesor ahora, debe de pasar de ser un mero transmisor a facilitador, guía; compartiendo el protagonismo del proceso de enseñanza con el alumno. La utilización de las TIC supone, sin duda una mejora en el proceso E - A, si el profesor sabe integrar las TIC en planteamientos didácticos y concreciones metodológicas significativas para el aprendizaje. Nos estamos refiriendo a metodologías que contemplen la autonomía del alumno para aprender, que permitan trabajar destrezas interpersonales y cognitivas fundamentales (Gimeno, 2002). 
Tabla 1. Competencias en TIC del profesorado

\begin{tabular}{|l|l|}
\hline Autor & Competencias en TIC del profesorado \\
\hline Salinas (1999) & $\begin{array}{l}\text { 1. Guiar a los alumnos en la información y conocimiento, } \\
\text { proporcionando accesos a los mismos para usar sus propios } \\
\text { recursos } \\
\text { 2. Potenciar en los alumnos su propio proceso de } \\
\text { aprendizaje } \\
\text { 3. Asesorar y gestionar el ambiente de aprendizaje en el que } \\
\text { los alumnos están utilizando estos recursos } \\
\text { 4. Acceder fluido en el trabajo del estudiante de acuerdo } \\
\text { con las estrategias de aprendizaje empleadas. }\end{array}$ \\
\hline Cabero (2000) & $\begin{array}{l}\text { 1. Organizador, diseñador y evaluador de situaciones } \\
\text { mediadas de aprendizaje } \\
\text { 2. Diseñador de medios adaptados a las características de } \\
\text { sus estudiantes y potencialidades de la tecnología utilizada. } \\
\text { 3. Interaccionar adecuadamente en entornos diferentes de } \\
\text { formación presencial cara a cara. } \\
\text { 4. Poseer cierto dominio tecnológico } \\
\text { 5. Poseer habilidades para trabajar en equipo, que } \\
\text { conlleva la capacidad de organizar de forma dinámica el } \\
\text { currículo estableciendo y adoptando criterios para la } \\
\text { creación de entornos colaborativos para el aprendizaje. }\end{array}$ \\
\hline
\end{tabular}




\begin{tabular}{|c|c|}
\hline Adell y Sales (2000) & $\begin{array}{l}\text { 1. Diseñar el currículo de acuerdo a los nuevos } \\
\text { escenarios educativos } \\
\text { 2. Potenciador de las funciones de autorización y } \\
\text { facilitador del aprendizaje. } \\
\text { 3. Elaborar materiales usando las posibilidades ofertadas: } \\
\text { digitalización y multimedia. }\end{array}$ \\
\hline Adell (1997) & $\begin{array}{l}\text { 1. Facilitador, guía y consejero sobre fuentes } \\
\text { apropiadas de información } \\
\text { 2. Creador de hábitos y destrezas en la búsqueda, } \\
\text { selección y tratamiento de la información } \\
\text { 3. Utilizar sus conocimientos y destrezas como } \\
\text { Herramientas al servicio de su propia autoformación. }\end{array}$ \\
\hline Gisbert (2000) & $\begin{array}{l}\text { 1. } \text { Consultor de la información } \\
\text { 2. Colaborador en grupo } \\
\text { 3. Facilitador } \\
\text { 4. } \\
\text { 5. Suparrollador de materiales } \\
\end{array}$ \\
\hline Marqués (2001) & $\begin{array}{l}\text { 1. Conocer y saber usar aparatos y programas } \\
\text { informáticos de uso general } \\
\text { 2. Conocer y utilizar bases de datos y programas } \\
\text { informáticos específicos } \\
\text { 3. Aplicar las TIC a la enseñanza como instrumento de } \\
\text { innovación didáctica. } \\
\text { 4. Actualizarse tecnológicamente } \\
\text { 5. Elaborar y mantener páginas web }\end{array}$ \\
\hline
\end{tabular}




\section{CONCLUSIONES}

Los medios y recursos posibilitan una mejor calidad en los procesos de enseñanza aprendizaje, con la irrupción e inclusión de las Tecnologías en la educación, las posibilidades educativas se ven incrementadas con el componente sociocultural y convierte a la alfabetización digital (multimodal) en un imperativo ético y de ciudadanía con entidad propia.

Bautista utiliza el término de alfabetización multimodal para definir la relación armónica entre tres formas de representar la realidad: textual, audiovisual y digital, que nos permiten el uso de los productos tecnológicos actuales. Este término que nos ayuda a comprender que los espacios multimedia, proporcionan a quien los utilizan el conocimiento y dominio de diversos lenguajes, ampliando su competencia lectora a través de lo que hemos denominado lectura multimodal y, por tanto, también su competencia para comunicar y expresarse de un modo creativo y colaborativo. Es decir, las TIC nos brinda nuevas formas de generar, comunicar y comprender contenidos significativos por medio de textos codificados bajo formatos diversos (Bautista, 2007).

La incorporación de la tecnología al curriculum puede favorecer el desarrollo personal del alumnado, al igual que alfabetizar supone aprender a leer y escribir, la alfabetización digital implica recibir y producir información, con unos denominadores comunes a ambas alfabetizaciones: la crítica (leer / recibir información) y la creatividad (escritura / producción de información). De acuerdo con Freire (1973 y 1984) alfabetizar no consiste en enseñar a repetir palabras escritas para conquistar cierta soltura mecánica, ni trasmitir sucesos o informaciones, quizás ya pasadas; a los alumnos, por el contrario debemos enseñarles cómo decir sus palabras, cómo escribir sucesos contextualizados y de su interés, ganar distancia de los acontecimientos, de la experiencia vivida, para analizarla y representarla en un texto. En definitiva, como nos plantea el autor, concienciar, tomar distancia crítica de la situación de dominación, desarrollar una práctica de liberación o libertad transformando críticamente la realidad y considerando a los hombres y mujeres como agentes de dicha transformación.

En los espacios de formación y tras nuestro proceso de indagación, extraemos como conclusiones y como necesidad el compromiso y una nueva forma de alfabetización, A. Bautista (2007) lo denomina alfabetización multimodal o una alfabetización como necesidad politica y moral (Área, 2001) que proporcione al profesorado una formación sobre la relación sociedad-tecnologías-escuela desde una dimensión sociocrítica sobre la presencia de estas tecnologías en los contextos sociales. Esta formación debe servir de instrumento que oriente sus prácticas docentes con las tecnologías. 
Las tecnologías en educación debieran ir más allá, queremos decir que desde este trabajo y en coincidencia con el Consejo de Europa (1996), resultaría imprescindible, hacer conscientes que el conocimiento y uso de las tecnologías no puede ser un fin en sí mismo, sino un medio para conocer mejor la sociedad y poder preparar a los alumnos para ser felices en ella: Los profesores deben aumentar sus conocimientos sobre las tendencias mundiales a fin de mejorar la orientación de su enseñanza. La evolución del mundo actual no se limita a la introducción de las tecnologías, sino que incluye los distintos fenómenos asociados a ellas, las rápidas transformaciones del mercado del trabajo, la creciente movilidad que se exige de la población trabajadora y las tendencias del desempleo y la consiguiente reorganización de la jornada laboral. El desafío de las tecnologías debe ser asumido por un sistema educativo que ofrezca a todos una sólida educación general y una buena formación profesional.

\section{REFERENCIAS}

Adell, J. (1997). Tendencias en educación en la sociedad de las tecnologías de la información Edutec. Revista electrónica de tecnología educativa, 7.

Area Moreira, M. (Coord.) (2001). Educar en la Sociedad de la Información. Bilbao: Desclée De Brouwer.

Bautista García-Vera, A. (1994). Las nuevas tecnologías en la capacitación docente. Madrid. Visor.

Bautista, A. (1994). El papel de los intelectuales y la no neutralidad de la tecnología: razones para un uso crítico de los recursos en la enseñanza. Revista de educación, 303: 243-260.

Blanco, N. (2005). Innovar más allá de las reformas: Reconocer el saber de la escuela. Revista Electrónica Iberoamericana sobre Calidad, Eficacia y Cambio en Educación, $3(1)$.

Cabero Almenara J. (1998). Impacto de las nuevas tecnologías de la información y la comunicación en las organizaciones educativas. En Enfoques en la organización y dirección de instituciones educativas formales y no formales, Granada: Grupo Editorial Universitario.

Carbonell, J. (2001). La aventura de innovar. Madrid. Morata.

Carr, W; Kemmis, S. (1988). Teoría Crítica de la Enseñanza. La Investigación-acción en la Formación del profesorado. Barcelona: Martínez Roca. 
Castells, M. (1997). La era de la información. Vol. 2. El poder de la identidad. Madrid: Alianza Editorial.

Castells, M. (1998). La era de la información. Vol. 3. Fin de Milenio. Madrid: Alianza Editorial.

Foucault, M. (1970). La arqueología del saber. México: Siglo XXI.

Freire, P. (1970). La Pedagogía del oprimido. Méjico: Siglo XX.

Freire, P. (1998): Pedagogía de la autonomía. Madrid: Siglo XXI Editores.

Freire, P. (1990). La naturaleza política de la educación. Barcelona: Cultura, poder y liberación.

Fullan, M. (1994). La gestión basada en el centro: el olvido de lo fundamental. Revista de Educación, 304: 147-161.

Fullan, M. (2002a). Liderar en una cultura de cambio. Barcelona: Octaedro.

Fullan, M. (2002b). Los nuevos significados del cambio en la educación. Barcelona.

Octaedro.

Gimeno Sacristán, J. (1998). Poderes inestables en educación. Madrid: Morata.

Gimeno Sacristán, J. (2005). La educación que aún es posible. Madrid: Morata.

Gimeno Sacristán, J. (2006). La reforma necesaria: entre la política educativa y la práctica escolar. Madrid: Morata.

Hargreaves, A. (1999). Schools and the Future: the Key Role of Innovation in: Innovation Schools. Centre for Educational Research and Innovation. Paris, OECD.

Hargreaves, A et al. (2001) Aprender a cambiar. La enseñanza más allá de las materias y los niveles. Barcelona: Octaedro.

Latapí, P. (1997). Tiempo educativo mexicano IV. México: Universidad Autónoma de México.

OECD (1999). "Education Policy Analysis". Centre for Educational Research and Innovation. Paris, OECD. 
Rayón Rumayor, L. (2000). Sobre mitos tecnológicos, proclamas totalizadoras, y alternativas educativas. Revista Kikiriki, 57: 4-16.

Rayón Rumayor, L. \& Rodríguez Torres, J. (2006). La necesaria <<voz>> del docente para la integración curricular de las TIC. OGE, 4.

Salinas, J. (1999): Formación y tecnologías de la información y la comunicación. En Noves tecnologies a la formació. Consellería de Treball i Benestar Social., Palma de Mallorca.

San Martín, A. (2004). La escuela enredada. Formas de participación escolar en la sociedad de la Información. Barcelona: Gedisa.

Tejada, J. (1998). Los agentes de la innovación en los Centros Educativos (Profesores, directivos y asesores). Granada: Ed. Aljibe.

\section{Javier Rodríguez-Torres}

Doctor en Pedagogía por la Universidad de Alcalá, Licenciado en Pedagogía y Diplomado en Magisterio por la Universidad Complutense de Madrid, Máster en Psicología Escolar por la misma Universidad. Más de veinte años en la Enseñanza no universitaria, ejerciendo como Profesor de Pedagogía Terapéutica, Profesor de Diversificación Curricular, Idioma (francés), Orientador de E. Infantil y Primaria. Desde el curso 2004 Profesor en la Universidad de Castilla - La Mancha. En la actualidad, Secretario del Departamento. Autor de varios artículos en revistas y capítulos de libros, premios nacionales, autonómicos y provinciales a distintos proyectos de investigación e innovación educativa. 